\title{
Evaluation of Various Methods for Detecting Biofilm Formation among the Clinical Isolates of Staphylococcus species
}

\author{
Shruti Nair ${ }^{1}$ and S. Megha ${ }^{2 *}$ \\ ${ }^{1}$ Adichunchanagiri Institute of Medical Sciences, B.G. Nagar, Mandya-571448, India \\ ${ }^{2}$ Department of Microbiology, Adichunchanagiri Institute of Medical Sciences, B.G. Nagar, \\ Mandya-571448, India \\ *Corresponding author
}

\section{A B S T R A C T}

\begin{tabular}{l} 
K e y w o r d s \\
Biofilm, tissue \\
culture plate \\
method, congo red \\
agar, tube \\
adherence, \\
Staphylococcus \\
Article Info \\
\hline $\begin{array}{l}\text { Accepted: } \\
\text { 18 May 2020 } \\
\text { Available Online: } \\
\text { 10 June } 2020\end{array}$ \\
\hline
\end{tabular}

\section{Introduction}

Biofilm is an assemblage of microbial cells that is irreversibly associated with a surface and enclosed in a matrix of polysaccharide material. It is composed primarily of microbial cells and extracellular polymeric substance (EPS)(CR Kokare et al., 2009). Adhesion to the surface provides advantages to the bacteria namely protection against microbial agents, acquisition of new genetic

\begin{abstract}
Biofilm producing strains of bacteria have become major cause for many chronic and persistent infections due to their increased antimicrobial resistance patterns. It is hence important to detect them as early as possible to administer appropriate treatment. The objective of this study is to compare the three conventionally used methods for biofilm detection, namely, Tissue culture plate, Tube adherence and Congo red agar method. 109 clinical isolates of Staphylococcus species were collected from various clinical samples and biofilm detection was checked for each by all of the above mentioned methods. Of these, 45 were identified as Staphylococcus aureus (SA) and 64 as Coagulase Negative Staphylococci (CONS). Positive biofilm producers by TCP method were $75(68.81 \%), 41(37.61 \%)$ by tube adherance method and $13(11.93 \%)$ by CRA method. Statistical analysis was done considering TCP method as gold standard and by using Chi square test. For tube adherence method sensitivity was $23.3 \%$, specificity $52.9 \%$ positive predictive value $60.9 \%$, negative predictive value $26.5 \%$ and p value was 0.171 . For CRA method sensitivity was $14.6 \%$, specificity $94.1 \%$ positive predictive value $84.6 \%$, negative predictive value $33.3 \%$ and $p$ value was 0.190 . TCP method was concluded to be the most sensitive, accurate and reproducible screening method among the three studied for detection of biofilm formation among Staphylococcal species.
\end{abstract}

traits, nutrient availability and metabolic cooperability (CR Kokare et al., 2009).Biofilm associated bacteria are less susceptible to antibiotics than planktonic bacteria; this can be explained by different mechanisms such as the binding of antibiotics to biofilm components, reduced penetration of the antibiotic, slower growth of the bacteria, high bacterial density and altered gene expression in the bacteria (Pinheiro L et al., 2014; Stewart PS and Costerton JW, 2001). 
Staphylococci is the organism of choice for this project as they are most often associated with chronic infections of implanted medical devices (Dunne WM, 2002; Donlan RM, 2001; Raad I, 2000). The use of indwelling medical devices is important in the treatment of critically and chronically ill patients, however bacterial colonization of implanted foreign material can cause major medical and economic sequel. The increased use of indwelling medical devices has had considerable impact on the role of staphylococci in clinical medicine. Staphylococcus species are common in these cases, their major pathogenic factor being ability to form biofilm on polymeric surfaces (Kloos WE and Bannerman TL, 1994), allowing adherence to biomedical implants and protection from host immune system and antimicrobial therapy (O'Gara and Humphreys, 2001).

Biofilms are notoriously difficult to eradicate and are often resistant to systemic antibiotic therapy and removal of infected device becomes necessary (Lewis, 2001; Schwank S, 1998; Souli and Giamarellou, 1998). Hence, testing forbiofilm formation is important in deciding the pathogenicity of various Staphylococcus species and should be routinely performed in diagnostic laboratories (Shrestha, et al., 2018). In light of the same, the objectives of this study include to isolate various Staphylococcus species from clinical specimens. And also to compare three phenotypic methods for detection of biofilm formation among the isolated Staphylococcus species, namely, Tube adherence method, Tissue culture plate method and Congo red agar method.

\section{Materials and Methods}

The present study was conducted in the Department of Microbiology, Adichunchanagiri Institute of Medical
Sciences, B.G. Nagara.A comparative study was conducted for a period of two months from $1^{\text {st }}$ June 2019 to $31^{\text {st }}$ July 2019.

A total of 109 Staphylococcal isolates obtained from various clinical samples like blood, pus, sputum, high vaginal swab and urine from patients of Adichunchanagiri Hospital and Research Centre, were included in the study.

\section{Inclusion criteria}

All the Staphylococcus species isolated were included.

\section{Exclusion criteria}

Gram negative organisms and Gram positive organisms other than Staphylococcus species were excluded.

Isolation and identification of Staphylococcus species was done according to CLSI guidelines (CLSI, 30 ${ }^{\text {th }}$ Edition).Institutional Ethical Committee (EIC) clearancewas obtained.

Samples collected were streaked onto Nutrient agar plates and incubated at $37^{\circ} \mathrm{C}$ for 18-24 hrs under aerobic culture conditions. The plates were then be examined for Staphylococcus colonies on the basis of their morphological aspects (creamy, yellow, whiteor greyish, convex, circular, smooth, shiny, opaque and easily emulsifiable colonies) (Ananthanarayan and Panicker, $9^{\text {th }}$ Edition). The pure isolates were then subjected to biochemical differentiation tests and isolates were characterized.

All the isolates were subjected to three methods for detecting biofilm production, namely, Tube adherence method, Tissue culture plate method and Congo red agar method. 


\section{Tube adherence method}

A loopful of colony suspension from an overnight culture was inoculated into trypticase soy broth medium and incubated for 24 hours at $35^{\circ} \mathrm{C}$. Then, the tubes were inverted and washedwith phosphate buffered saline of $\mathrm{pH}$ 7.3. After drying, they were stained with $0.1 \%$ crystal violet. The tubes were rinsed multiple times with running tap water to remove excess stain. The tubes were kept in an inverted position and the tubes showing retention of the crystal violet were considered positive for biofilm formation (Christensen GD et al., 1982).

\section{Tissue culture plate method}

This method, proposed by Christensen et al., is the gold standard method for detection of biofilm formation. The isolates were inoculated in TSB medium and incubated at $37^{\circ} \mathrm{C}$ for $18-24$ hours in aerobic conditions. Then, the individual wells of 96 well TCPs were filled with 100 microlitre of culture suspension. After overnight incubation, the plates were tapped gently for the removal of the free flowing bacteria and washed four times with PBS. The plates were air dried, fixed and stained with $0.1 \%$ crystal violet for 5 minutes. Then the plates were rinsed under running tap water several times to remove excess stain (Christensen GD et al., 1985).The results were read using TECAN multimode plate reader Infinite $\mathrm{M} \mathrm{Nano}^{+}$by absorbance method at $590 \mathrm{~nm}$ wavelength.

\section{Congo red agar method}

This method was proposed by Freeman et al., The Congo Red Agar was prepared by mixing $37 \mathrm{~g}$ brain heart infusion broth, $50 \mathrm{~g}$ sucrose, $0.8 \mathrm{~g}$ Congo red dye and $10 \mathrm{~g}$ agar in 1 litre of distilled water. The isolates were streaked on the CRA and incubated aerobically at $35^{\circ} \mathrm{C}$ for 18-24 hours. The strains producing black and dry colonies were identified as biofilm producers and those producing red colonies as non biofilm producers (Freeman DJ et al., 1989).

All the procedures were carried out in accordance with universal precaution in triplicate form.

\section{Results and Discussion}

A total of 109 isolates of Staphylococci were obtained from different clinical samples. Out of these, $45(41.28 \%)$ were Staphylococcus aureus (SA) and 64 (58.71\%) Coagulase Negative Staphylococci (CONS). Positive biofilm producers by TCP method were $75(68.81 \%) \quad[31 \quad(28.44 \%) \quad \mathrm{SA}$ and 44 (40.37\%) CONS]. By tube adherence method, $41(37.61 \%)$ isolates were detected as positive and $13(11.93 \%)$ were positive by CRA method (Figure 1,2).

Among the positive producers, maximum came from pus samples (40), followed by blood (13) and urine (12). (Table 1)

Antimicrobial resistance patterns clearly show that the positive biofilm producers had higher resistance to most antibiotics (but no resistance seen against Vancomycin and Linezolid )than non-producers as determined by AST against Penicillin, Ampicillin, Gentamicin, etc.(Figure 3) which was also seen in other studies (Bose S et al., 2009; Donlan and Costerton, 2002).

Among the 109 isolates, a total of $75(68.8 \%)$ were positive for biofilm formation by Tissue Culture Plate (TCP) method. 54(49.54\%) were detected as strong biofilm producers, 21(19.27\%) moderate, 13(11.92\%) weak and $21(19.27 \%)$ as non-producers. Strong and moderate producers were considered as positive. This segregation was done based on 
optical density(OD) values of the stained biofilm in the microtitre plate obtained by TECAN multimode plate reader Infinite $\mathrm{M}$ $\mathrm{Nano}^{+}$at $590 \mathrm{~nm}$ (OD value less than 0.1333 was considered non producer, 0.1333 to 0.2666 as weak, 0.2666 to 0.5332 as moderate and greater than 0.5332 as strong producers).(Figure 4) The cutoff OD was calculated by the following formula:

$\mathrm{OD}_{\text {cutoff }}=\mathrm{OD}_{\text {average }}$ of negative control $+(3$ $\mathrm{X}$ Standard Deviation of OD of negative control )

These results are similar to the findings of Knobloch et al., 2002), Nabajit Deka(2014) and Bose et al., 2009) in their respective studies. The percentages of biofilm producers is lesser as compared to the study by Deka $\mathrm{N}(2014)$ as we did not add glucose to the trypticase soy broth which further promotes biofilm production (Mathur T et al., 2006).

By Congo Red Agar (CRA) method, out of the 109 isolates, 13(11.93\%) were detected as positive biofilm producers. Strains producing black coloured dry colonies on the media were considered as positive producers and those producing red colonies were taken as negatives. (Figure 5)

These are consistent with the findings of Deka N(2014) and Bose et al., 2009). Results were similar to those from other studies as the Brain Heart Infusion media was sterilized separately before addition of the Congo red dye (Rania M et al., 2018).

The results from this method varied drastically from those obtained from tissue culture plate and tube adherence method. Of the 109 isolates, by Tube Adherence (TA) method, 41(37.61\%) were positive biofilm producers of which $8(7.34 \%)$ were strong and $33(30.27 \%)$ were moderate producers
(Figure 6), which is similar to readings obtained by Taj Y et al., 2012) and Bose et al., 2009). Though the results compared well with those from TCP method, it was difficult to differentiate between strong and moderate biofilm producers since interpretation would be subjective, which is the case in the study by Mathur et al., 2006) as well.

Statistical analysis was done using chi square test considering tissue culture plate method as gold standard. Congo red agar method showed 2 false positives and 64 false negatives, whereas with tube adherence method, 16 were false positives and 50 false negatives.(Table 2,3,4) Test with $\mathrm{P}$ value $\leq$ 0.05 is considered reliable. The comparative analysis yielded the following results:

Tube adherence method showed 16 false positives and 50 false negatives. Sensitivity was $33.3 \%$, specificity $52.9 \%$ positive predictive value $60.9 \%$, negative predictive value $26.5 \%$ and $P$ value was 0.171 by Chi square test. Accuracy was $39.4 \%$. In the study by Mathur T et al., 2006) sensitivity is $77.9 \%$ and specificity $96 \%$.

Congo red agar method showed 2 false positives and 64 false negatives.

Sensitivity was $14.6 \%$, specificity $94.1 \%$ positive predictive value $84.6 \%$, negative predictive value $33.3 \%$ and $\mathrm{P}$ value was 0.190 by Chi square test. Accuracy was $39.4 \%$ (Table. 2,3,4). In the study by Mathur, et al., 2006) sensitivity was $7.6 \%$ and specificity $97.2 \%$.

Though tissue culture plate method was considered gold standard, it is very labour intensive and expensive as compared to the other two. Test results have to be confirmed with genotypic methods. 
Table.1 Biofilm producers among different clinical specimens

\begin{tabular}{|l|c|c|c|}
\hline Sample & Positive & Negative & Total \\
\hline Pus & 40 & 15 & 55 \\
\hline Blood & 13 & 5 & 18 \\
\hline Urine & 12 & 12 & 24 \\
\hline Sputum & 2 & 0 & 2 \\
\hline HVS & 5 & 1 & 6 \\
\hline Other & 3 & 1 & 4 \\
\hline Total & 75 & 34 & 109 \\
\hline
\end{tabular}

Table.2 Statistical analysis for Congo red agar method considering TCP as gold standard

\begin{tabular}{|l|l|c|c|c|c|}
\hline \multicolumn{2}{|c|}{} & \multicolumn{2}{|c|}{ TCP } & Total & $\begin{array}{c}\text { P value } \\
\text { (Chi square test) }\end{array}$ \\
\cline { 3 - 6 } & Positive & Negative & & 0.190 \\
\hline $\begin{array}{l}\text { Congo } \\
\text { Red Agar } \\
\text { Method }\end{array}$ & Positive & 11 & 2 & 13 & 96 \\
\hline Total & Negative & 64 & 32 & 109 & \\
\hline
\end{tabular}

Table.3 Statistical analysis for Tube adherence method considering TCP as gold standard

\begin{tabular}{|l|l|c|c|c|c|}
\hline \multirow{2}{*}{} & \multicolumn{2}{|c|}{ TCP } & Total & $\begin{array}{c}\text { P value } \\
\text { (Chi square } \\
\text { test) }\end{array}$ \\
\cline { 2 - 5 } & Positive & Negative & & \multirow{2}{*}{0.171} \\
\hline $\begin{array}{l}\text { Tube } \\
\text { Adherence } \\
\text { Method }\end{array}$ & Positive & 25 & 16 & 41 & \\
\cline { 1 - 5 } Total & Negative & 50 & 18 & 109 & \\
\hline
\end{tabular}

Table.4 Sensitivity and specificity of Congo red agar and Tube adherence method considering TCP as gold standard

\begin{tabular}{|l|c|c|c|c|c|}
\hline & Sensitivity & Specificity & PPV & NPV & Accuracy \\
\hline CRA & 14.6 & 94.1 & 84.6 & 33.3 & 39.4 \\
\hline TAM & 33.3 & 52.9 & 60.9 & 26.5 & 39.4 \\
\hline
\end{tabular}




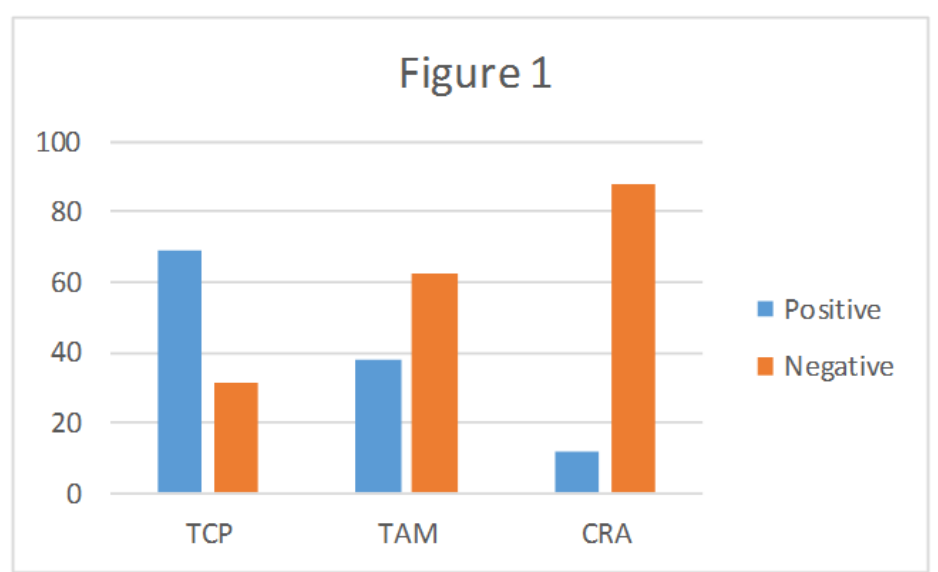

Figure.1 Detection of biofilm formation in clinical isolates of Staphylococcal species by TCP (tissue culture plate), TA (tube adherence) and CRA (congo red agar) methods

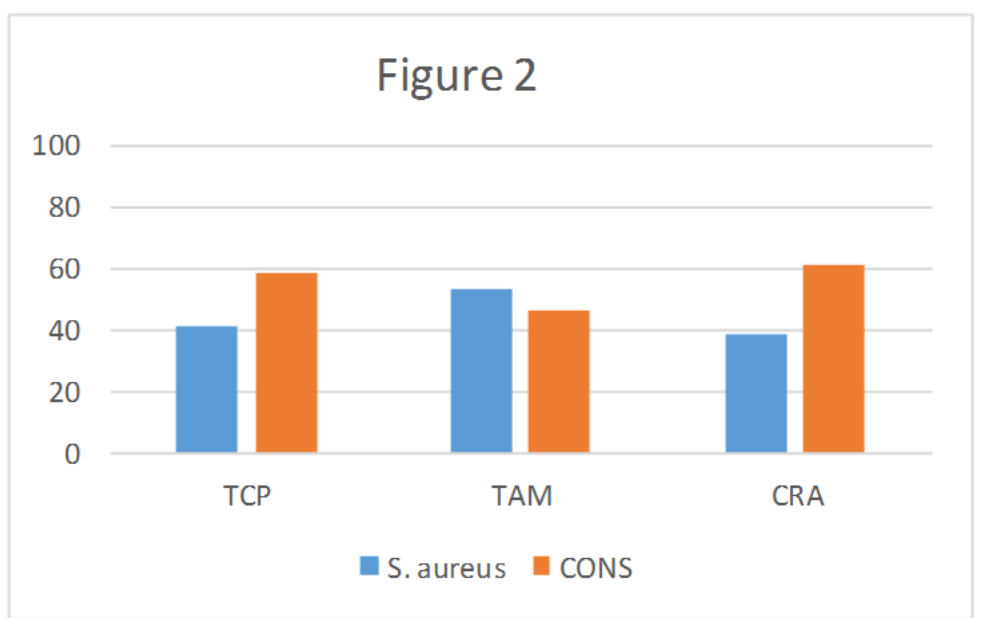

Figure.2 Segregation of positive biofilm producers as $S$. aureus and CONS by TCP (tissue culture plate), TA (tube adherence) and CRA (congo red agar) methods

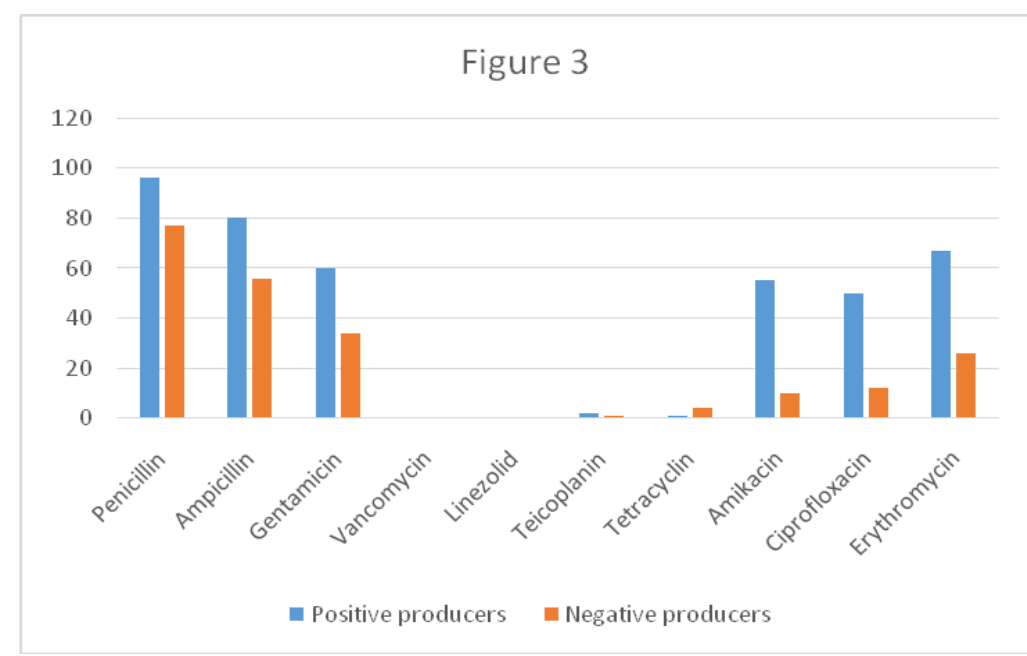

Figure.3 Comparison of antimicrobial resistance patterns of biofilm producers with that of non-biofilm producers 


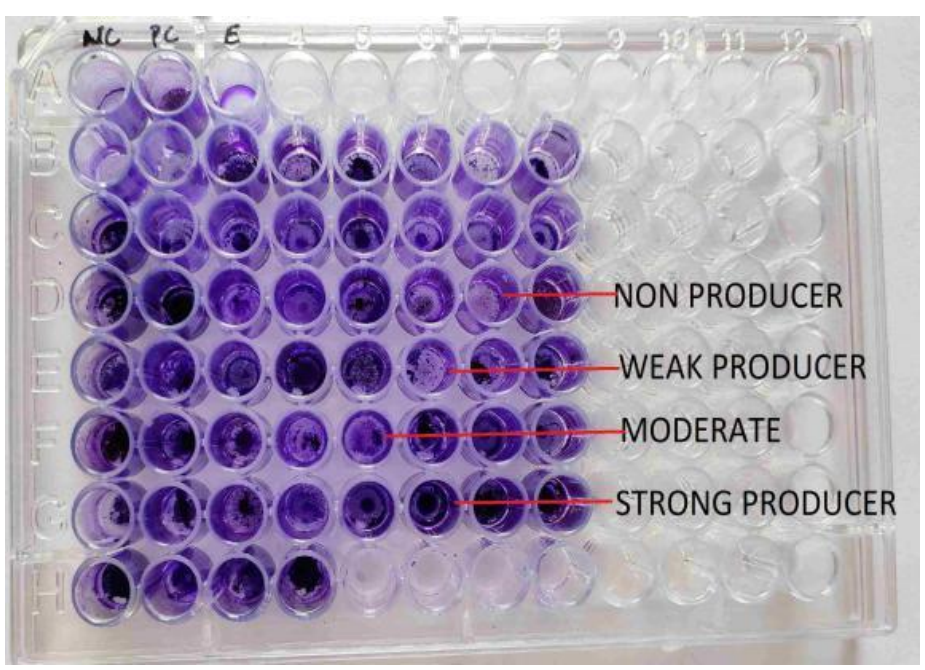

Figure.4 Biofilm detection by Tissue Culture Plate method. The figure shows strong, moderate, weak and non-biofilm producers

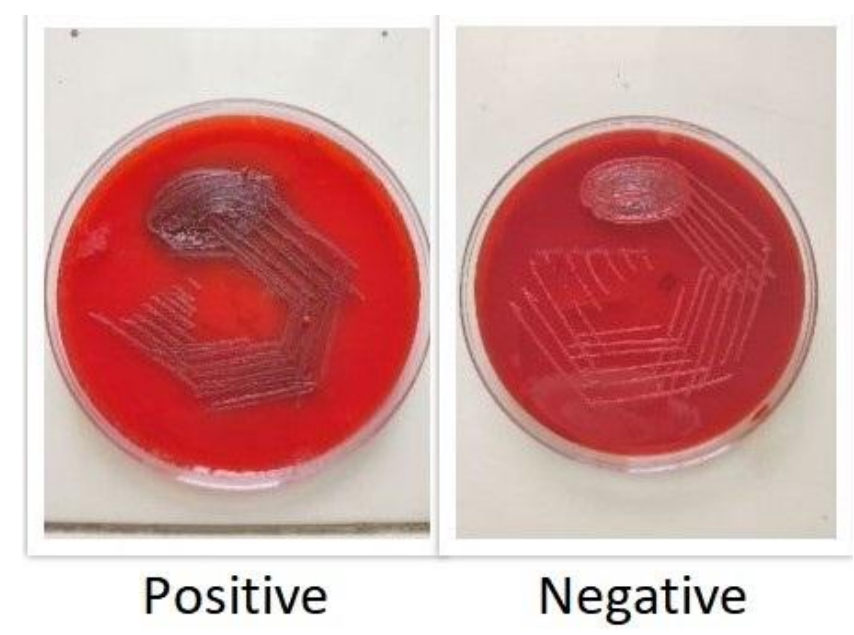

Figure.5 Congo red agar media showing biofilm producer (black) and non biofilm producer (red)

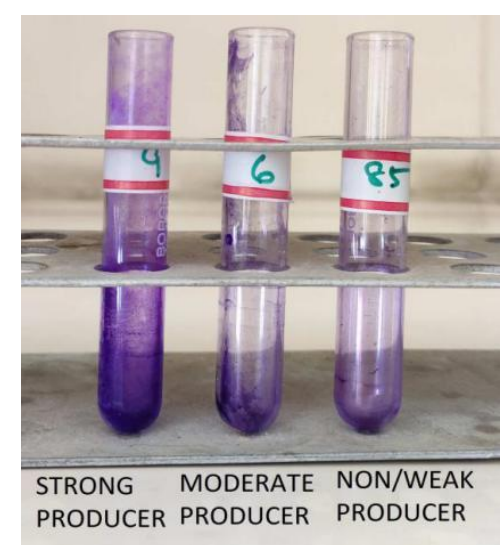

Figure.6 Biofilm detection by Tube Adherence method showing strong, moderate and weak producers 
Tube adherence method has sensitivity greater than congo red agar method, but results vary greatly as the result interpretation is subjective. Congo red agar method is the cheapest of the three and the best screening method, but has very low sensitivity.

All these are phenotypic methods and can be biased. Genotypic methods will give more accurate results but they, along with the newer methods such as confocal laser scanning microscopy, RNAseq, microarrays and RT-qPCR are expensive and difficult to perform in routine laboratories and are better as confirmatory tests (Franklin MJ et al., 2015; Oliveira A et al., 2010). Hence reliable, convenient and inexpensive methods are needed to screen for biofilm formation which are accessible to most diagnostic laboratories, particularly those located in resource limited countries (Goyal R et al., 2006).

In conclusion, TCP method was found to be the most sensitive, accurate and reproducible screening method for detection of biofilm formation among Staphylococcal species. Tube adherence method had better sensitivity than Congo red agar method. The definitive confirmatory method would be genotypic study, though it could be expensive.

\section{Acknowledgement}

This Research project was made possible due to support from Dr MG Shivaramu, Principal Adichunchanagiri Institute of Medical Sciences and Dr Venkatesha D, Professor and HOD, Dept. Of Microbiology, AIMS, for which I am forever grateful. I am also thankful to Dr Megha S, Assisstant Professor, Dept. Of Microbiology, AIMS, for providing her guidance and wisdom through the course of this project. I also wish to extend my gratitude to Dr Sudhanva, AIMS and the faculty and members of the Department of Microbiology and the Central Research
Institute, AIMS, for providing their ever important assistance.

\section{References}

Ananthanarayan and Panicker, Textbook of Microbiology, $9^{\text {th }}$ Edition. 49-53, 199207, 635-639.

Bose S, Khodke M, Basak S, Mallick S K. Detection of biofilm producing Staphylococci: Need of the hour. J Clin Diagn Res. 2009;3:1915-20.

C R Kokare, S Chakraborty, A N Khopade, et al., Biofilm: Importance and applications. Ind J Biotech. 2009;8:15968.

Christensen G D, Simpson W A, Bisno A L, et al., Adherence of slime producing strains of Staphylococcus epidermidis to smooth surfaces. Infect Immun. 1982;37(1):318-26.

Christensen G D, Simpson W A, Younger J J, et al., Adherence of coagulase negative staphylococci to plastic tissue culture plates: a quantitative model for the adherence of staphylococci to medical devices. $J$ Clin Microbiol. 1985;22(6):996-1006.

Clinical and Laboratory Standard Institute. Performance standards for antimicrobial susceptibility testing; M100, $30^{\text {th }}$ edition.,2020.

Deka N. Comparison of Tissue Culture plate method, Tube Method and Congo Red Agar Method for the detection of biofilm formation by Coagulase Negative Staphylococcus isolated from Non-clinical Isolates. Int $J$ Curr Microbiol App Sci. 2014;3(10):810-15.

Donlan R M, Costerton W. Biofilms: Survival mechanisms of clinically relevant microorganisms. Clin Microbiol Rev. 2002;15(2):167-93.

Donlan RM. Biofilms and device associated infections. Emerg Infect Dis. 2001;7:277-81.

Dunne WM. Bacterial adhesion: Seen any good biofilms lately? Clin Microbiol Rev. 
2002;15:155-66.

Franklin M J, Chang C et al., New technologies for studying biofilms. Microbiol Spectr. 2015;3(4).

Freeman D J, Falkiner F R, Keane C T. New method for detecting slime production by coagulase negative staphylococci. $J$ Clin Pathol. 1989;42(8):872-74.

Goyal R, Singh N P et al., Simple and economical method for speciation and resistotyping of clinically significant coagulase negative staphylococci. Indian $J$ Med Microbiol. 2006;24(3):201-204.

Kloos WE, Bannerman TL. Update on clinical significance of coagulase-negative Staphylococci. Clin Microbiol Rev. 1994;7:117-40.

Knobloch_J K, Horstkotte M A,Rohde_H, Mack_D. Evaluation of different detection methods of biofilm formation in Staphylococcus aureus. Med Microbiol Immunol. 2002;191(2):1016.

Lewis K. Riddle of biofilm resistance. Antimicrob Agents Chemother. 2001;45:999-1007.

Mathur T, Singhal S, Khan S, Upadhyay D J, Fatma T, Rattan A. Detection of biofilm formation among the clinical isolates of staphylococci: An evaluation of three different screening methods. Indian $J$ Med Microbiol. 2006;24:25-9.

O'Gara JP, Humphreys H. Staphylococcus epidermidis biofilms : Importance and implications. $J$ Med Microbiol. 2001;50:582-7.

Oliveira A, Cunha Mde L et al., Comparison of methods for the detection of biofilm production in coagulase negative
Staphylococci. BMC Res Notes. 2010;3:260.

Pinheiro L, Brito C I, Pereira V C, et al., Reduced susceptibility to vancomycin and biofilm formation in methicillin resistant Staphylococcus epidermidis isolated from blood cultures. Mem Inst Oswaldo Cruz. 2014;109(7):871-78.

Raad I. Management of intravascular catheterrelated infections. $J$ Antimicrob Chemother. 2000;45:267-70.

Rania M, Abdel Halim et al., Detection of Biofilm producing Staphylococci among different clinical isolates and its relation to Methicillin susceptibility. Maced J Med Sci. 2018;6(8):1335-41.

Schwank S, Rajacic Z, Zimmerli W, Blaser J. Impact of bacterial biofilm formation on in vitro and in vivo activities of antibiotics. Antimicrob Agents Chemother. 1998;42:895-8.

Shrestha L B, Bhattarai N R, Khanal B.Comparative evaluation of methods for detection of biofilm formation in coagulase negative staphylococci and correlation with antibiogram. Inf Drug Res. 2018;11:607-13.

Souli M, Giamarellou H. Effects of slime production by clinical isolates of coagulase negative staphylococci on activities of various antimicrobial agents. Antimicrob Agents Chemother. 1998;42:939-41.

Stewart P S, Costerton J W. Antibiotic resistance of bacteria in biofilms. Lancet. 2001;358(9276):135-38.

Taj Y, Essa F, Aziz F, Kazmi S U.Study on biofilm-forming properties of clinical isolates of Staphylococcus aureus. J Infect Dev Ctries. 2012;6(5):403-9.

\section{How to cite this article:}

Shruti Nair and Megha. S. 2020. Evaluation of Various Methods for Detecting Biofilm Formation among the Clinical Isolates of Staphylococcus Species. Int.J.Curr.Microbiol.App.Sci. 9(06): 2195-2203. doi: https://doi.org/10.20546/ijcmas.2020.906.268 